FIAN/TD-14/11

ITEP/TH-24/11

\title{
Integrability properties of Hurwitz partition functions. II. Multiplication of cut-and-join operators and WDVV equations
}

\author{
A.Mironov ${ }^{*}$ A.Morozov ${ }^{\dagger}$ and S.Natanzon ${ }^{\ddagger}$
}

\begin{abstract}
Correlators in topological theories are given by the values of a linear form on the products of operators from a commutative associative algebra (CAA). As a corollary, partition functions of topological theory always satisfy the generalized WDVV equations of [33]. We consider the Hurwitz partition functions, associated in this way with the CAA of cut-and-join operators. The ordinary Hurwitz numbers for a given number of sheets in the covering provide trivial (sums of exponentials) solutions to the WDVV equations, with finite number of time-variables. The generalized Hurwitz numbers from [26] provide a non-trivial solution with infinite number of times. The simplest solution of this type is associated with a subring, generated by the dilatation operators $\hat{W}_{1}=\operatorname{tr} D=\operatorname{tr} X \partial / \partial X$.
\end{abstract}

\section{Introduction}

Hurwitz theory is one of the rapidly developing branches of modern mathematical physics [1]-[27]. It has its origins in the enumeration problem of ramified coverings of $C P^{1}$, and it is brought into modern context with the formula due to Frobenius, [3]

$$
\operatorname{Cover}_{n}\left(\Delta_{1}, \ldots, \Delta_{m}\right)=\sum_{R} d_{R}^{2} \varphi_{R}\left(\Delta_{1}\right) \ldots \varphi_{R}\left(\Delta_{m}\right) \delta_{|R|, n}
$$

expressing the covering multiplicities through the quantities $\varphi_{R}(\Delta)$ proportional to the characters of the symmetric group $S_{n}$ [28]. Here $\Delta_{i}$ is the Young diagram (integer partition), characterizing the type (conjugation class) of ramification point, all sizes of the diagrams being the same and equal to the number of sheets in the covering, $\left|\Delta_{1}\right|=\ldots=\left|\Delta_{m}\right|=n$, and the sum runs over the Young diagrams $R$ of the same size $|R|=n$, but this time they label representations of the symmetric group. The symmetric group characters are among the most important objects in combinatorics, more sophisticated then the $G L(\infty)$ characters $\chi_{R}(t)$, very well-known and used in physical applications [29]. Still the two sets of characters are directly related by the Frobenius formula, which is a sort of Fourier transform [28],

$$
\chi_{R}(t)=\sum_{\Delta} d_{R} \varphi_{R}(\Delta) p(\Delta) \delta_{|\Delta|,|R|}
$$

where $\Delta=\left[\ldots \geq \delta_{2} \geq \delta_{1}\right]=\left[\ldots, 3^{m_{3}}, 2^{m_{2}}, 1^{m_{1}}\right]$, its size $|\Delta|=\sum_{k} \delta_{k}=\sum_{k} k m_{k}$, the time monomial

$$
p(\Delta)=\prod_{k} p_{\delta_{k}}=\prod_{k} p_{k}^{m_{k}}
$$

and $p_{k}=k t_{k}$. These two character sets are further unified through the concept [8] of cut-and-join operators $\hat{W}(\Delta)$, commuting differential operators of degree $|\Delta|$ in $t$-variables, for which they serve as eigenvectors and eigenvalues respectively $[26]$ :

$$
\hat{W}(\Delta) \chi_{R}(t)=\varphi_{R}(\Delta) \chi_{R}(t)
$$

\footnotetext{
*Lebedev Physics Institute and ITEP, Moscow, Russia; mironov@itep.ru; mironov@lpi.ru

$\dagger$ ITEP, Moscow, Russia; morozov@itep.ru

$\ddagger$ Department of Mathematics, Higher School of Economics, Moscow, Russia; ITEP, Moscow, Russia and A.N.Belozersky Institute, Moscow State University, Moscow, Russia; natanzons@mail.ru
} 
The problem with these operators is that they look rather complicated (they belong to the class of $W$-operators, made from powers of the $U(1)$ current). In [26] we explained that the cut-and-join operators acquire a very simple form if expressed in terms of the matrix Miwa variables $p_{k}=\operatorname{tr} X^{k}$, then ${ }^{1}$

$$
\hat{W}_{\Delta}=: \prod_{k} \frac{1}{k^{m_{k}} m_{k} !}\left(\operatorname{tr} \hat{D}^{k}\right)^{m_{k}}:
$$

for the $G L(\infty)$ matrix generator $D=X \frac{\partial}{\partial X_{\mathrm{tr}}}$. This representation opens a constructive way to evaluation of the structure constants $C_{\Delta_{1} \Delta_{2}}^{\Delta}$, which appear in the CAA of cut-and-join operators and, as a consequence, in the ordinary multiplication algebra of symmetric group characters (this algebra was earlier considered from combinatorial point of view in [30] where it is claimed to be equivalent to the algebra of shifted symmetric functions of [31]):

$$
\begin{array}{r}
\hat{W}_{\Delta_{1}} \hat{W}_{\Delta_{2}}=\sum_{\Delta} C_{\Delta_{1} \Delta_{2}}^{\Delta} \hat{W}_{\Delta}, \\
\varphi_{R}\left(\Delta_{1}\right) \varphi_{R}\left(\Delta_{2}\right)=\sum_{\Delta} C_{\Delta_{1} \Delta_{2}}^{\Delta} \varphi_{R}(\Delta)
\end{array}
$$

It is important in these formulae that the sums over $\Delta$ are not restricted to $|\Delta|=\left|\Delta_{1,2}\right|$, moreover, $\left|\Delta_{1}\right|$ can be different from $\left|\Delta_{2}\right|$. Note that already in (4) there is no restriction to $|R|=|\Delta|$, and $\varphi_{R}(\Delta)$ 's in this formula are more general than those in (2). They are defined for the Young with $r$ unit rows, $[\Delta]=\left[\tilde{\Delta}, 1^{r}\right]$ by adding additional unit rows to reach $|R|=|\Delta|$ in accordance with the rule

$$
\varphi_{R}([\Delta]) \equiv\left\{\begin{array}{ccc}
0 & \text { for } & |\Delta|>|R| \\
\frac{(|R|-|\Delta|+r) !}{r !(R|-| \Delta \mid) !} \varphi_{R}([\Delta, \underbrace{1, \ldots, 1}_{|R|-|\Delta|}]) & \text { for } & |\Delta| \leq|R|
\end{array}\right.
$$

This naturally leads to extension of (1) by removing the projector $\delta_{|R|, n}$ and lifting the restriction $\left|\Delta_{1}\right|=\ldots=$ $\left|\Delta_{m}\right|=n$, which defines what was called generalized Hurwitz numbers in [26].

Note that the CAA of cut-and-join operators induces the multiplication on the Young diagrams:

$$
\Delta_{1} * \Delta_{2}=\sum_{\Delta} C_{\Delta_{1} \Delta_{2}}^{\Delta} \Delta
$$

This multiplication can be considered as extension of another o-multiplication on the Young diagrams, given by the composition of permutations and related to the ordinary Hurwitz numbers. This latter is connected with the *-multiplication by restricting onto diagrams of the same size:

$$
\Delta_{1} \circ \Delta_{2}=\sum_{\Delta} C_{\Delta_{1} \Delta_{2}}^{\Delta} \Delta \delta_{\left|\Delta_{1}\right|,|\Delta|}
$$

with $\left|\Delta_{1}\right|=\left|\Delta_{2}\right|$. Inversely, one can construct $*$-multiplication from the o-one by the procedure described in section 3 .

In this paper we discuss one of the immediate implications of the extension to the generalized Hurwitz numbers: their generating function is the partition function of a topological field theory associated with the CAA (6) and, hence, satisfies the WDVV equations of [33]. The ordinary Hurwitz partition functions, based on the ordinary o-multiplication in symmetric group $S(n)$, also satisfy WDVV for each given $n$, but they provide only trivial solutions (which, however, were not discussed in the existing literature). The WDVV equations are imposed on "quasiclassical $\tau$-functions", which are obtained by one or another kind of Whitham averaging procedure [34] from the KP/Toda hierarchy and a particular set of Riemann surfaces (background) with additional data. The quasiclassical hierarchies are well studied in the case when the background is a Riemann sphere, but in the case of Hurwitz theory it should be different (a Lambert curve, for example, $[21,22,19]$ ), and such hierarchies are not yet described (see, however, [35]). An advantage of the quasiclassical hierarchy would be that particular equations involve only derivatives w.r.t. the finite number of time-variables, while the WDVV equations involve inversion of an infinite size matrix. Derivation of such reducible equations for Hurwitz partition functions remains an open problem.

\footnotetext{
${ }^{1}$ The combinatorial coefficient $z(\Delta)=\prod_{k} \frac{1}{k^{m} k m_{k} !}$ here that counts the order of the automorphism group of the Young diagram, appears everywhere in the theory of symmetric functions and symmetric group $S(\infty)$. In particular, the standardly normalized symmetric group characters $\hat{\chi}_{R}(\Delta)=d_{R} z(\Delta) \varphi_{R}(\Delta)$. They are generated by the command $C h i(R, \Delta)$ in Maple in the package combinat.
} 
This paper is the second in the series which describes properties of the generating functions in Hurwitz theory. The first paper, [27] contains a summary of integrable properties, which will be described in detail in our next paper, [32].

In section 2 we begin with reminding the general construction of topological theory for any CAA and explain why its partition function always satisfy the WDVV equations (their original form is a little more general than in [33], and far more general than in [36], the triple derivative equations of [33] being a direct corollary, but not vice versa!). Then, in sections 3 we provide discuss the two multiplications: $\circ$ and ${ }^{*}$-products. The corresponding multiplication tables can be found in Appendices I and II. Knowledge of these tables allows one to examine concrete examples illuminating following sections. In section 4 we construct two types of generating functions which are associated with two multiplications, and in section 5 the corresponding WDVV equations satisfied by these generating functions are discussed. As a particular example, in sections 6 and 7 we provide details about the $\left[1^{p}\right]$ subring of the ${ }^{*}$-algebra, describing cut-and-join operators, associated with the single row diagrams (the "complementary" single column operators would instead generate the entire algebra).

\section{Topological theories and WDVV}

\subsection{Topological theory on sphere (tree level)}

At the tree (string) level (i.e. on sphere) a topological theory is defined by three ingredients:

- a vector space with the basis of "observables" $\left\{\phi_{i}\right\}$,

- an associative and commutative multiplication

$$
\phi_{i} * \phi_{j}=\sum_{k} C_{i j}^{k} \phi_{k}
$$

- and a linear form (c-valued function) on this space $\left.<\phi_{i}\right\rangle=K_{i}$.

At the loop (string) level (i.e. on higher genera Riemann surfaces) one needs also to define traces and impose an additional constraint on the toric 1-point function (in addition to associativity and commutativity of the multiplication), but we do not need this in the present text, which is fully devoted to the tree level topological theories.

The tree correlators are defined as

$$
K_{i_{1} \ldots i_{n}}=<\phi_{i_{1}}, \ldots, \phi_{i_{n}}>=<\phi_{i_{1}} * \ldots * \phi_{i_{n}}>=\sum_{k} C_{i_{1} \ldots i_{n}}^{k}<\phi_{k}>=\sum_{k} C_{i_{1} \ldots i_{n}}^{k} K_{k}
$$

where the coefficients $C$ are products of the original 3-valent structure constants $C_{i j}^{k}$. These correlators are totally symmetric under permutations of $i_{1}, \ldots, i_{n}$.

It is also convenient to introduce "the bare metric"

$$
G_{i j} \equiv<\phi_{i}, \phi_{j}>=<\phi_{i} * \phi_{j}>=\sum_{k} C_{i j}^{k}<\phi_{k}>
$$

and use it and its inverse $G^{i j}$ to raise and lower indices, in particular, to construct the totally symmetric tensors

$$
C_{i j k} \equiv<\phi_{i}, \phi_{j}, \phi_{k}>=\sum_{m} C_{i j}^{m} G_{m k}=\sum_{m} C_{j k}^{m} G_{m i}=\sum_{m} C_{i k}^{m} G_{m j}
$$

and

$$
<\phi_{i}, \phi_{j}, \phi_{k}, \phi_{l}>=\sum_{m} C_{i j}^{m} C_{m k l}=\sum_{m} C_{i k}^{m} C_{m j l}=\sum_{m} C_{i l}^{m} C_{m j k}=\ldots
$$

Next one defines the tree partition function

$$
Z[\beta]=\left\langle e_{*}^{\sum_{i} \beta_{i} \phi_{i}}\right\rangle=<E[\beta]>\equiv<<1>>
$$

where $e_{*}(\phi)=\sum_{n} \frac{1}{n !} \underbrace{\phi * \phi * \ldots * \phi}_{n}$. Then,

$$
C_{i j k}=\left.\frac{\partial^{3} Z[\beta]}{\partial \beta_{i} \partial \beta_{j} \partial \beta_{k}}\right|_{\beta=0}
$$




\subsection{Deformation by coupling constants $\beta$ and WDVV equations}

One can now introduce deformed, $\beta$-dependent algebra with a $\beta$-dependent multiplication

$$
\phi_{i} \hat{*} \phi_{j} \equiv \phi_{i} * \phi_{j} * E[\beta] \equiv \sum_{k} \hat{C}_{i j}^{k} \phi_{k}
$$

where $E[\beta]$ is a family of elements of the algebra. The new multiplication is still commutative and associative:

$$
\left(\phi_{i} \hat{*} \phi_{j}\right) \hat{*} \phi_{k}=\phi_{i} * \phi_{j} * \phi_{k} * E[\beta] * E[\beta]=\phi_{i} \hat{*}\left(\phi_{j} \hat{*} \phi_{k}\right)
$$

It is also possible to introduce the deformed observables $\hat{\phi}_{i}=\phi_{i} * E[\beta]$, then

$$
\hat{\phi}_{i} * \hat{\phi}_{j}=\phi_{i} * \phi_{j} * E[\beta] * E[\beta]=\left(\phi_{i} \hat{*} \phi_{j}\right) * E[\beta]=\left(\widehat{\phi_{i} \hat{*} \phi_{j}}\right)=\sum_{k} \hat{C}_{i j}^{k} \hat{\phi}_{k}
$$

is also a commutative associative algebra.

Now one can introduces the $\beta$-dependent correlators:

$$
<<\phi_{i_{1}}, \ldots, \phi_{i_{n}}>>\equiv<\phi_{i_{1}} * \ldots * \phi_{i_{n}} * E[\beta]>
$$

Then the triple correlator possesses two alternative representations (the last two sums in this formula):

$$
\hat{C}_{i j k} \equiv \frac{\partial^{3} Z[\beta]}{\partial \beta_{i} \partial \beta_{j} \partial \beta_{k}}=<<\phi_{i}, \phi_{j}, \phi_{k}>>=<\phi_{i} * \phi_{j} * \phi_{k} * E[\beta]>=\sum_{m} \hat{C}_{i j}^{m} G_{m k}=\sum_{m} C_{i j}^{m} \hat{G}_{m k}
$$

The first representation is in terms of deformed $\hat{C}_{i j}^{k}$ and the bare metric $G_{m k}=<\phi_{k} * \phi_{m}>$, while the second one is in terms of the bare (undeformed) $C_{i j}^{k}$ and the deformed metric

$$
\hat{G}_{m k} \equiv<<\phi_{m}, \phi_{k}>>=<\phi_{m} * \phi_{k} * E[\beta]>=\frac{\partial^{2} Z[\beta]}{\partial \beta_{k} \partial \beta_{m}}
$$

Associativity of original and deformed algebras is expressed in the commutativity condition of the structure constants $\left(\check{C}_{i}\right)_{j}^{k} \equiv C_{i j}^{k}$

$$
\check{C}_{i} \check{C}_{j}=\check{C}_{j} \check{C}_{i}
$$

In its turn, this implies that

$$
\hat{C}_{i j m} G^{m n} \hat{C}_{k l n}=\hat{C}_{i k m} G^{m n} \hat{C}_{j l n}
$$

and

$$
\hat{C}_{i j m} \hat{G}^{m n} \hat{C}_{k l n}=\hat{C}_{i k m} \hat{G}^{m n} \hat{C}_{j l n}
$$

which we respectively call as bare and full $[33,38]$ WDVV equations for the partition function $Z[\beta]$ (in the case of the full WDVV equation all ingredients are triple derivatives of $Z[\beta]$, the deformed metric is the triple correlator with the unity operator $\left.\phi_{0}=I: \hat{G}_{m n}=\hat{C}_{0 m n}\right)$.

In some cases the choice of the vector space and observables $\phi_{i}$ is not unique. The same algebra may possess different realizations (representations) and one can ask if the $\beta$-deformed topological theory respects this freedom. The problem is very similar to the representation theory of Lie algebras and to the concept of the universal group elements etc.

\subsection{Hurwitz topological theory}

Consider here an explicit example when $\phi_{i}$ has an additional label, $R$ that is the variable averaged in the mean value $\langle\ldots\rangle$, and such that the structure constants in the product

$$
\phi_{i}(R) \phi_{j}(R)=\sum_{k} C_{i j}^{k} \phi_{k}(R)
$$


do not depend on $R$. An example of such topological theory is provided by the theory of Hurwitz numbers, and the role of index $R$ can be played by different structures, for instance, the Young diagrams in the Frobenius formula (1). In this case, we define the correlators involving the sum over $R$ :

$$
<i_{1}, \ldots, i_{n}>=<\phi_{i_{1}}(R) * \ldots * \phi_{i_{n}}(R)>=\sum_{R} d_{R}^{2} \phi_{i_{1}}(R) \ldots \phi_{i_{n}}(R)
$$

Now, it is clear that the equality

$$
\begin{array}{r}
\hat{C}_{i j k} \equiv<<i, j, k>>=<\phi_{i}(R) * \phi_{j}(R) * \phi_{k}(R) * E[\beta, R]>= \\
=\sum_{m} C_{i j}^{m}<\phi_{m}(R) * \phi_{k}(R) * E[\beta, R]>=\sum_{m} C_{i j}^{m} \hat{G}_{m k}=\sum_{m} \hat{C}_{i j}^{m} G_{m k}
\end{array}
$$

with

$$
E[\beta, R]=e_{*}^{\sum_{i} \beta_{i} \phi_{i}(R)}
$$

continues to hold in this case.

As follows from the discussion above, the Hurwitz partition function as a function of $\beta$ satisfies the full WDVV equations (25). Sometime, for restricted sets of $\beta$-variables, it happen to be also KP $\tau$-functions [27] but this is beyond our consideration here. Instead we note that the weight $E[\beta, R]$ can be made more general than (29), without changing anything in the content of the previous consideration. Namely, one can change (29) for

$$
P_{*}(\phi(R)) * e_{*}^{\sum_{i} \beta_{i} \phi_{i}(R)}
$$

with an arbitrary *-polynomial of observables $\phi_{i}(R)$ with the same $R$. Sometimes new integrability properties can occur for the partition function as a function on these additional parameters [27].

In Hurwitz theory per se, the role of observables $\phi_{i}(R)$ is played by the characters of the symmetric group $S_{\infty}$, denoted by $\varphi_{R}(\Delta)$, where the label $i \rightarrow \Delta$ is now the Young diagram. The most interesting choice for $P_{*}(\phi(R))$ is a product of several $G L(\infty)$ characters, $\chi_{R}(t) \chi_{R}\left(t^{\prime}\right) \chi_{R}\left(t^{\prime \prime}\right) \ldots$, where $\chi_{R}(t)$ is related to $\varphi_{R}(\Delta)$ by formula (2). With so modified $E[\beta, R]$ the Hurwitz partition function becomes a function of both $t$ - and $\beta$ variables. While in $\beta$-variables (when considering their complete set, not a subset) it is usually a "quasiclassical $\tau$-function", i.e. a solution to the full WDVV equations, in $t$-variables it can be a KP $\tau$-function. This is, indeed, the case when there is one $(t)$ and two $\left(t, t^{\prime}\right)$ sets of $t$-variables, see [39, 9, 27,32]. Surprisingly or not, the KP integrability in $t$ disappears for three $\left(t, t^{\prime}, t^{\prime \prime}\right)$ or more sets of $t$-variables. This peculiar pattern of (non)-integrability structures is discussed in the next paper of this series [32].

In variance with our construction in this section, in [37] there was suggested another, polynomial class of solutions to the WDVV equations. In the Hurwitz theory context this would correspond to a power series instead of polynomial solutions.

\section{Two multiplications of Young diagrams}

As we mentioned above, there are two natural multiplications on the Young diagrams: one, o-multiplication given by the composition of permutations, and the other one, *-multiplication induced by the algebra of cutand-join operators (5).

\section{1 o-Multiplication of Young diagrams from composition of permutations}

The o-multiplication is given on the Young diagrams (integer partitions) labeling elements of the group algebra of the symmetric group, i.e. the sum of all permutations ${ }^{2}$ from the corresponding conjugation class:

$$
[211]=(12)+(13)+(14)+(23)+(24)+(34)
$$

etc. The number of items is denoted by $\|\Delta\|$.

The naive o-multiplication of Young diagrams, induced by the (non-commutative but associative) composition of permutations is commutative and associative. Of course, $\left\|\Delta_{1} \circ \Delta_{2}\right\|=\left\|\Delta_{1}\right\| \cdot\left\|\Delta_{2}\right\|$.

Examples of the multiplication tables for different symmetric groups can be found in Appendix II.

\footnotetext{
${ }^{2}$ The composition of permutations is done in Maple by the command mulperms of the package group.
} 
- For any $k$ the o-multiplication by $\left[1^{k}\right]$ acts like unity:

$$
\left[1^{k}\right] \circ \Delta=\Delta \quad \forall \Delta:|\Delta|=k
$$

- Multiplication by $\left[2,1^{k}\right]$ can be deduced from the cut-and-join property. Namely, if permutations are written in the cyclic notations, then permutation $(12) \in S_{k+2}$ acts as follows:

$$
\begin{aligned}
&(12) \circ(12) K=K, \\
&(12) \circ(12 C) K=(1 C) K,(12) \circ(21 C) K=(2 C) K, \\
&(12) \circ(1 C) K=(12 C) K,(12) \circ(C) K=(12)(C) K, \\
&(12) \circ\left(1 C_{1}\right)\left(2 C_{2}\right) K=\left(1 C_{2} 2 C_{1}\right) K
\end{aligned}
$$

where $C$ denotes any set of elements, and $K$ any set of non-intersecting cycles (of course, it is assumed that $1,2 \notin C, C_{1}, C_{2}, K$ and $\left.C, C_{1}, C_{2} \notin K\right)$. If for a given level $k$ all the "powers" $\|\Delta\|$ are known, this is enough to construct all the entries $\left[2,1^{k}\right] \circ \Delta$ in the o-multiplication table: the coefficient for each line in (32) is given by the ratio of items of the given type at the l.h.s. of (32) to the "power" at the r.h.s., multiplied by $\left\|2,1^{k}\right\|$. These rules are illustrated in manifest examples of Appendix I.

\section{$3.2 *$-multiplication of Young diagrams}

The *-multiplication of Young diagrams is associated with the product of the differential cut-and-join operators (5): if

$$
\hat{W}\left[\Delta_{1}\right] \hat{W}\left[\Delta_{2}\right]=\sum_{\Delta} C_{\Delta_{1} \Delta_{2}}^{\Delta} \hat{W}[\Delta]
$$

then

$$
\Delta_{1} * \Delta_{2}=\sum_{\Delta} C_{\Delta_{1} \Delta_{2}}^{\Delta} \Delta
$$

and thus it is commutative and associative. The sums are actually finite: the size of the diagrams $\Delta$ is restricted to

$$
\max \left(\left|\Delta_{1}\right|,\left|\Delta_{2}\right|\right) \leq|\Delta| \leq\left|\Delta_{1}\right|+\left|\Delta_{2}\right|
$$

As it was already mentioned in the Introduction, these commuting $\hat{W}$-operators have all the $G L(\infty)$ characters as common eigenfunctions, while $S_{\infty}$ characters are the corresponding eigenvalues (4). Representations of $G L(\infty)$ characters through the first and second Weyl formulas are associated to representations of the cut-andjoin operators in time and matrix variables. It follows from (4) that the symmetric group characters form the same commutative associative algebra:

$$
\varphi_{R}\left(\Delta_{1}\right) \varphi_{R}\left(\Delta_{2}\right)=\sum_{\Delta} C_{\Delta_{1} \Delta_{2}}^{\Delta} \varphi_{R}(\Delta)
$$

with the same $R$-independent structure constants $C_{\Delta_{1} \Delta_{2}}^{\Delta}$.

\subsection{Connection between the two multiplications}

The *-multiplication (34) can be expressed through the o-multiplication of Young diagrams. It is a rather long recursive formula, but actually a very constructive one:

$$
\begin{gathered}
\Delta_{1} * \Delta_{2}=\sum_{n=\max \left(\left|\Delta_{1}\right|,\left|\Delta_{2}\right|\right)}^{\left|\Delta_{1}\right|+\left|\Delta_{2}\right|}\left\{\Delta_{1}, \Delta_{2}\right\}_{n} \\
\left\{\Delta_{1}, \Delta_{2}\right\}_{n}=\sum_{\Delta:|\Delta|=n} C_{\Delta_{1} \Delta_{2}}^{\Delta} \Delta=\rho_{n-\left|\Delta_{1}\right|}\left(\Delta_{1}\right) \circ \rho_{n-\left|\Delta_{2}\right|}\left(\Delta_{2}\right)-\sum_{k=\max \left(\left|\Delta_{1}\right|,\left|\Delta_{2}\right|\right)}^{n-1} \rho_{n-k}\left(\left\{\Delta_{1}, \Delta_{2}\right\}_{k}\right)
\end{gathered}
$$


and $\rho_{k}$ is a lift of the Young diagram to the size $|\Delta|+k$, achieved by adding $k$ unit length rows with additional numeric factor: if $\Delta$ already has $r$ rows of the length 1 , then

$$
\rho_{k}([\Delta])=\frac{(r+k) !}{r ! k !}[\Delta, \underbrace{1, \ldots, 1}_{k}], \quad \text { or } \quad \rho_{k}\left(\left[\tilde{\Delta}, 1^{r}\right]\right)=\frac{(r+k) !}{r ! k !}\left[\tilde{\Delta}, 1^{r+k}\right]
$$

where $[\Delta] \equiv\left[\tilde{\Delta}, 1^{r}\right]$ and $\tilde{\Delta}$ does not contains unit rows. According to this definition, $\rho_{0}(\Delta)=\Delta$. Note that

$$
\rho_{k}\left(\rho_{l}(\Delta)\right)=\frac{(k+l+r) !}{k ! l ! r !}\left[\Delta, 1^{k+l}\right] \quad \neq \quad \rho_{k+l}(\Delta)=\frac{(k+l+r) !}{(k+l) ! r !}\left[\Delta, 1^{k+l}\right]
$$

The highest term in the product (37) is

$$
\left\{\Delta_{1}, \Delta_{2}\right\}_{\left|\Delta_{1}\right|+\left|\Delta_{2}\right|}=C_{\Delta_{1} \Delta_{2}}^{\left[\Delta_{1}, \Delta_{2}\right]}\left[\Delta_{1}, \Delta_{2}\right]
$$

and for $\Delta_{1}=\left[k^{m_{k}}\right], \Delta_{2}=\left[k^{n_{k}}\right],\left[\Delta_{1}, \Delta_{2}\right]=\left[k^{m_{k}+n_{k}}\right]$ the combinatorial coefficient is

$$
C_{\Delta_{1} \Delta_{2}}^{\left[\Delta_{1}, \Delta_{2}\right]}=\prod_{k} \frac{\left(m_{k}+n_{k}\right) !}{m_{k} ! n_{k} !}
$$

This follows from the definition (5) of the $\hat{W}$ operator [26].

If $\Delta_{1} * \Delta_{2}=\sum_{\Delta} C_{\Delta_{1} \Delta_{2}}^{\Delta} \Delta$, formula (37) can be rewritten as

$$
\rho_{n-|\Delta|_{1}}\left(\Delta_{1}\right) \circ \rho_{n-|\Delta|_{2}}\left(\Delta_{2}\right)=\sum_{m} \rho_{n-m}\left(\left\{\Delta_{1}, \Delta_{2}\right\}_{m}\right)=\sum_{\Delta} C_{\Delta_{1} \Delta_{2}}^{\Delta} \rho_{n-|\Delta|}(\Delta)
$$

Expressed in terms of the generating functions $J_{\Delta}(u)=\sum_{m=0}^{\infty} u^{|\Delta|+m} \rho_{m}(\Delta)$ this multiplication formula becomes

$$
\oint J_{\Delta_{1}}(u) \circ J_{\Delta_{2}}\left(\frac{v}{u}\right) \frac{d u}{u}=\sum_{\Delta} C_{\Delta_{1} \Delta_{2}}^{\Delta} J_{\Delta}(v)=J_{\Delta_{1} * \Delta_{2}}(v)
$$

Note that the contour integral over $u$ at the l.h.s. selects diagrams of the same weight, so that the operation $\circ$ is well defined.

Examples of the *-multiplication tables can be found in section 6 and in Appendix II, here we consider only the case of product $[1] *[\Delta]$ which will be of use for our further consideration.

\subsection{Example of level $(1, m)$}

For $\Delta$ of the size $|\Delta|=m$, which already has $r$ columns of height 1 , one gets

$$
\begin{gathered}
{[1] *[\Delta]=\{1, \Delta\}_{m}+\{1, \Delta\}_{m+1},} \\
\{1, \Delta\}_{m}=\rho_{m-1}[1] \circ \Delta=m\left[1^{m}\right] \circ \Delta=m \Delta, \\
\{1, \Delta\}_{m+1}=\rho_{m}[1] \circ \rho_{1}[\Delta]-\rho_{1}\left(\{1, \Delta\}_{m}\right)=(m+1)\left[1^{m+1}\right] \circ(r+1)[\Delta, 1]-m(r+1)[\Delta, 1]=(r+1)[\Delta, 1], \\
{[1] * \Delta=|\Delta| \Delta+(r+1)[\Delta, 1]}
\end{gathered}
$$

In other words, if $\Delta=\left[\tilde{\Delta}, 1^{r}\right]$, where $\tilde{\Delta}$ contains no more units, then

$$
[1] *\left[\tilde{\Delta}, 1^{r}\right]=(|\tilde{\Delta}|+r)\left[\tilde{\Delta}, 1^{r}\right]+(r+1)\left[\tilde{\Delta}, 1^{r+1}\right]
$$

\section{Generating functions}

Introduce now a linear form (average) on the Young diagrams:

$$
<\Delta>=\frac{\delta\left(\Delta,\left[1^{|\Delta|}\right]\right)}{|\Delta| !}=\sum_{R} d_{R}^{2} \varphi_{R}(\Delta) \delta_{|R|,|\Delta|}
$$

It can be used to construct a variety of generating functions for averages of Young diagrams products (correlators).

In fact, the projection to $|R|=|\Delta|$ in the sum over $R$ in (46) can be eliminated, and the infinite sum

$$
\sum_{R} d_{R}^{2} \varphi_{R}(\Delta)=\sum_{R:|R| \geq|\Delta|} d_{R}^{2} \varphi_{R}(\Delta)=e<\Delta>
$$

where $e=2.718 \ldots$ This formula is important for evaluation of the partition functions in the case of *-products. 


\subsection{The standard Hurwitz partition function}

The standard generating function of Hurwitz numbers is

$Z_{\circ}\left\{\beta_{\tilde{\Delta}}\right\}=\sum_{n} q^{n} Z_{\circ}^{(n)}\left\{\beta_{\Delta}\right\}=\sum_{n} q^{n}\left\langle\exp _{\circ}\left(\sum_{\tilde{\Delta}:|\tilde{\Delta}| \leq n} \beta_{\tilde{\Delta}} \rho_{n-|\tilde{\Delta}|}(\tilde{\Delta})\right)\right\rangle=\sum_{n} q^{n}\left\langle\exp _{\circ}\left(\sum_{\tilde{\Delta}:|\tilde{\Delta}| \leq n} \beta_{\tilde{\Delta}}\left[\tilde{\Delta}, 1^{n-|\Delta|}\right]\right)\right\rangle$

where, as before, $\tilde{\Delta}$ denotes the Young diagram without units and the o-multiplication of the Young diagram of different sizes is defined to be zero. Actually, $q=e^{\beta_{1}}$. Of course, one can also introduce a whole infinite tower of $\beta$-variables for each $\tilde{\Delta}: \beta_{\tilde{\Delta}, p}=\beta_{\left[\tilde{\Delta}, 1^{p}\right]}$, but we prefer not to do it. Again, for each given $n$ the component $Z_{\circ}^{(n)}\left\{\beta_{\Delta}\right\}$ satisfies the WDVV equations, but after summation over $n$, (48) is not an average of any CAA exponential and does not need to satisfy WDVV equations. And, indeed, it does not.

With the help of (2) one can rewrite (48) in terms of symmetric group characters $\varphi_{R}(\Delta),(7)$. Indeed, because of (4) they form a representation of the CAA algebra with *-product:

$$
\varphi_{R}\left(\Delta_{1}\right) \varphi_{R}\left(\Delta_{2}\right)=\varphi_{R}\left(\Delta_{1} * \Delta_{2}\right)=\sum_{\max \left(\left|\Delta_{1}\right|,\left|\Delta_{2}\right|\right) \leq|\Delta| \leq\left|\Delta_{1}\right|+\left|\Delta_{2}\right|} C_{\Delta_{1} \Delta_{2}}^{\Delta} \varphi_{R}(\Delta) \quad \forall R, \Delta_{1}, \Delta_{2}
$$

For $\left|\Delta_{1}\right|=\left|\Delta_{2}\right|=n$ and for $|R|=n$ the property (7) implies that also

$$
\varphi_{R}\left(\Delta_{1} \circ \Delta_{2}\right)=\varphi_{R}\left(\Delta_{1}\right) \varphi_{R}\left(\Delta_{2}\right), \text { for }|R|=\left|\Delta_{1}\right|=\left|\Delta_{2}\right|
$$

and this allows one to express $(48)$ through $\varphi_{R}(\Delta)$ : if all the sizes $\left|\Delta_{1}\right|=\ldots\left|\Delta_{k}\right|=n$ are the same, then

$$
\left\langle\mathrm{o}_{i=1}^{k} \Delta_{i}\right\rangle=\sum_{\Delta} c_{\Delta_{1}, \ldots, \Delta_{k}}^{\Delta}<\Delta>\delta_{|\Delta|, n}=\sum_{\Delta} c_{\Delta_{1}, \ldots, \Delta_{k}} \sum_{R} d_{R}^{2} \varphi_{R}(\Delta) \delta_{|\Delta|, n} \delta_{|R|, n}=\sum_{R} d_{R}^{2} \prod_{i=1}^{k} \varphi_{R}\left(\Delta_{i}\right) \delta_{|R|, n}
$$

Here

$$
c_{\Delta_{1}, \ldots, \Delta_{k}}^{\Delta}=\sum_{\Delta_{1}^{\prime}, \ldots, \Delta_{k-2}^{\prime}} C_{\Delta_{1} \Delta_{1}^{\prime}}^{\Delta} C_{\Delta_{2} \Delta_{2}^{\prime}}^{\Delta_{1}^{\prime}} \ldots C_{\Delta_{k-1} \Delta_{k}}^{\Delta_{k-2}^{\prime}} \delta_{\left|\Delta_{1}^{\prime}\right|, n} \ldots \delta_{\left|\Delta_{k-2}^{\prime}\right|, n}
$$

we denote it by small letter $c$ to emphasize that all sums are restricted (projected) to diagrams of the same size $n$. Restriction to $|R|=n$ is important for the last transition in (51), where small $c$ stand at the l.h.s.: still equality takes place because of (50).

From (51) it follows directly that partition function (48) can be rewritten as

$$
Z_{\circ}\left\{\beta_{\tilde{\Delta}}\right\}=\sum_{n} q^{n} \sum_{R:|R|=n} d_{R}^{2} \exp \left(\sum_{\tilde{\Delta}:|\tilde{\Delta}| \leq n} \beta_{\tilde{\Delta}} \varphi_{R}\left(\tilde{\Delta}, 1^{n-|\tilde{\Delta}|}\right)\right)
$$

or simply

$$
Z_{\circ}\left\{\beta_{\Delta}\right\}=\sum_{n} q^{n} Z_{\circ}^{(n)}\left\{\beta_{\Delta}\right\}=\sum_{n} q^{n} \sum_{R:|R|=n} d_{R}^{2} \exp \left(\sum_{\Delta:|\Delta|=n} \beta_{\Delta} \varphi_{R}(\Delta)\right)
$$

where, in principle, one can either impose the restriction

$$
\beta_{\left[\tilde{\Delta}, 1^{r}\right]}=\beta_{\tilde{\Delta}}
$$

or not.

If (55) is not imposed, then, making use of (2), one can further perform a Fourier transform of its $m$-th derivative into $t$-variables:

$$
\begin{gathered}
\sum_{n} q^{n} \sum_{\substack{\Delta_{1}, \ldots, \Delta_{m} \\
\left|\Delta_{1}\right|=\ldots=\left|\Delta_{m}\right|=n}} \frac{\partial^{m} Z_{\circ}^{(n)}\left\{\beta_{\Delta}\right\}}{\partial \beta_{\Delta_{1}} \ldots \partial \beta_{\Delta_{m}}} p^{(1)}\left(\Delta_{1}\right) \ldots p^{(m)}\left(\Delta_{m}\right)= \\
=\sum_{n} q^{n} \sum_{R:|R|=n} d_{R}^{2-m} \chi_{R}\left(t^{(1)}\right) \ldots \chi_{R}\left(t^{(m)}\right) \exp \left(\sum_{\tilde{\Delta}:|\tilde{\Delta}|=n} \beta_{\tilde{\Delta}} \varphi_{R}(\tilde{\Delta})\right)
\end{gathered}
$$




\subsection{Extension to *-product}

With the ${ }^{*}$-product one can associate another, generalized Hurwitz partition function [26]:

$$
Z_{*}\left\{\beta_{\Delta}\right\}=\left\langle\exp _{*}\left(\sum_{\Delta} \beta_{\Delta} \Delta\right)\right\rangle
$$

In variance with $Z_{\circ}\left\{\beta_{\Delta}\right\}$ in (48), it satisfies the WDVV equations, but in variance with individual components $Z_{\circ}^{(n)}\left\{\beta_{\Delta}\right\}$ (which also satisfy WDVV) it involves infinitely many time-variables $\beta_{\Delta}$.

One can also rewrite $Z_{*}$ in terms of $\varphi_{R}(\Delta)$ characters, but this time, in the case of *-products, there would be no restriction on the sizes $|\Delta|$ in (51). Then, in this case the sum over $R$ will be restricted not to $|R|=n$, but to $|R|=|\Delta|$ :

$$
\left\langle *_{i=1}^{k} \Delta_{i}\right\rangle=\sum_{\Delta} C_{\Delta_{1}, \ldots, \Delta_{k}}^{\Delta}<\Delta>=\sum_{\Delta} C_{\Delta_{1}, \ldots, \Delta_{k}}^{\Delta} \sum_{R} d_{R}^{2} \varphi_{R}(\Delta) \delta_{|R|,|\Delta|}
$$

and, because of the $\Delta$-dependent projector, in this formula one can not make any direct use of the relation

$$
\sum_{\Delta} C_{\Delta_{1}, \ldots, \Delta_{k}}^{\Delta} \varphi_{R}(\Delta)=\prod_{i=1}^{k} \varphi_{R}\left(\Delta_{i}\right)
$$

However, one can actually get rid of the projector! The reason is that (2) has important generalizations:

$$
\begin{array}{r}
\sum_{\Delta} d_{R} \varphi_{R}(\Delta) p(\Delta) \delta_{|\Delta|,|R|}=\chi_{R}(t), \\
\sum_{\Delta} d_{R} \varphi_{R}(\Delta) p(\Delta)=\chi_{R}\left(t_{k}+\delta_{k 1}\right), \\
\sum_{\tilde{\Delta}} d_{R} \varphi_{R}(\tilde{\Delta}) p(\tilde{\Delta})=\chi_{R}\left(1, t_{2}, t_{3}, \ldots\right), \\
d_{R}=\chi_{R}(1,0,0, \ldots)=\chi_{R}\left(\delta_{k 1}\right)
\end{array}
$$

Note that because of the property (7) all the sums over $\Delta$ are finite, and these formulas are elementary, not transcendental.

Combining (61) and (63) with the celebrated Cauchy completeness formula

$$
\sum_{R} \chi_{R}(t) \chi_{R}\left(t^{\prime}\right)=\exp \left(\sum_{k} k t_{k} t_{k}^{\prime}\right)
$$

one obtains

$$
\sum_{R, \Delta} d_{R}^{2} \varphi_{R}(\Delta) p(\Delta)=\sum_{R} \chi_{R}\left(t_{k}+\delta_{k 1}\right) \chi_{R}\left(\delta_{k 1}\right)=e^{1+t_{1}}=e \sum_{\Delta}<\Delta>p(\Delta)=e \sum_{R, \Delta} d_{R}^{2} \varphi_{R}(\Delta) p(\Delta) \delta_{|R|,|\Delta|}
$$

In other words, we obtain the already-mentioned statement (47):

$$
<\Delta>=\sum_{R} d_{R}^{2} \varphi_{R}(\Delta) \delta_{|R|,|\Delta|}=\frac{1}{e} \sum_{R} d_{R}^{2} \varphi_{R}(\Delta)
$$

i.e. one can simply substitute everywhere the average (46) by the alternative one,

$$
<<\Delta>>=\sum_{R} d_{R}^{2} \varphi_{R}(\Delta)=e<\Delta>
$$

where sum goes over Young diagrams $R$ of all sizes, not restricted to $|R|=|\Delta|$. The difference is actually exhausted by a factor of $e=2.718 \ldots$ (59):

Coming back to (58), one now knows how to eliminate the unwanted projector from the r.h.s. and apply

$$
\left\langle *_{i=1}^{k} \Delta_{i}\right\rangle=\frac{1}{e}\left\langle\left\langle *_{i=1}^{k} \Delta_{i}\right\rangle\right\rangle=\frac{1}{e} \sum_{\Delta} C_{\Delta_{1} \ldots \Delta_{k}}^{\Delta}<\langle\Delta\rangle>=\frac{1}{e} \sum_{\Delta} C_{\Delta_{1} \ldots \Delta_{k}}^{\Delta} \sum_{R} d_{R}^{2} \varphi_{R}(\Delta)=\frac{1}{e} \sum_{R} d_{R}^{2} \prod_{i=1}^{k} \varphi_{R}\left(\Delta_{i}\right)
$$


In particular, the pair correlator is equal to

$$
\begin{aligned}
& \sum_{\Delta_{1}, \Delta_{2}}<\Delta_{1} * \Delta_{2}>p^{\Delta_{1}} \bar{p}^{\Delta_{2}}=\sum_{\Delta_{1}, \Delta_{2}} \frac{1}{e} \sum_{R} d_{R}^{2} \varphi_{R}\left(\Delta_{1}\right) \varphi_{R}\left(\Delta_{2}\right) p^{\Delta_{1}} \bar{p}^{\Delta_{2}} \stackrel{(61)}{=} \\
= & \frac{1}{e} \sum_{R} \chi_{R}\left(t_{k}+\delta_{k 1}\right) \chi_{R}\left(\bar{t}_{k}+\delta_{k 1}\right) \stackrel{(64)}{=} \exp \left(\left(t_{1}+1\right)\left(\bar{t}_{1}+1\right)-1+\sum_{k \geq 2} t_{k} \bar{t}_{k}\right)
\end{aligned}
$$

From formula (68) one obtains a character expansion of $Z_{*}$ and a much better counterpart of (56):

$$
Z_{*}\left\{\beta_{\Delta}\right\}=\left\langle\exp _{*}\left(\sum_{\Delta} \beta_{\Delta} \Delta\right)\right\rangle=\frac{1}{e} \sum_{R} d_{R}^{2} \exp \left(\sum_{\Delta} \beta_{\Delta} \varphi_{R}(\Delta)\right)
$$

and

$$
\begin{array}{r}
\sum_{\Delta_{1}, \ldots, \Delta_{m}} \frac{\partial^{m} Z_{*}\left\{\beta_{\Delta}\right\}}{\partial \beta_{\Delta_{1}} \ldots \partial \beta_{\Delta_{m}}} p^{(1)}\left(\Delta_{1}\right) \ldots p^{(m)}\left(\Delta_{m}\right)= \\
=\frac{1}{e} \sum_{n} q^{n} \sum_{R:|R|=n} d_{R}^{2-m} \chi_{R}\left(t_{k}^{(1)}+\delta_{k 1}\right) \ldots \chi_{R}\left(t_{k}^{(m)}+\delta_{k 1}\right) \exp \left(\sum_{\Delta} \beta_{\Delta} \varphi_{R}(\Delta)\right)
\end{array}
$$

We emphasize once again that there are no restrictions on the sizes of any $\Delta_{i}$ and of $R$ in the sum. In (71) we do not impose (55), otherwise one should just write correlators instead of $\beta$-derivatives at the l.h.s.

\section{$5 \quad$ WDVV equations}

Whenever the generating function is an average of the CAA exponential, it satisfies the WDVV equations. This happens, at least, in two cases. The first one is for the o-product at the given level $|\Delta|$ : then one gets a trivial WDVV solution in form of a finite linear combination of ordinary exponentials. The second case is that of the ${ }^{*}$-product: then the number of time-variables is infinite, even in the simplest case of the $\left[1^{p}\right]$-subring, an interesting open question being to find an adequate quasiclassical (dispersionless) hierarchy, which is associated with this particular solution to the WDVV equations: it is probably related to the KP-Whitham hierarchy over the Lambert curve $[21,22,19,35]$.

We start with the trivial case of the o-partition function, then comment on the case of the $*$-partition function when the WDVV equation are awaited on common grounds but one tediously check them directly. We postpone until the next two section a discussion of the case of $\left[1^{p}\right]$-subring when some less involved direct checks of the WDVV equations can be done.

\section{$5.1 \quad$ o-products at given level}

For a given $n$ one introduces a function of $\left|S_{n}\right|$ variables $\beta_{\Delta}$ :

$$
Z_{n}(\beta)=\left\langle\exp _{\circ}\left(\sum_{\Delta:|\Delta|=n} \beta_{\Delta} \Delta\right)\right\rangle=\sum_{R:|R|=n} d_{R}^{2} \prod_{\Delta:|\Delta|=n} e^{\beta_{\Delta} \varphi_{R}(\Delta)}
$$

Each $Z_{n}$ satisfies the set of WDVV equations. In this case this follows not only from the general arguments, true for any topological field theory, but also from a much simpler consideration: one can obtain by a linear transform from $\left\{\beta_{\Delta}\right\}$ the separated variables

$$
\xi_{R}=\sum_{\Delta:|\Delta|=n} \beta_{\Delta} \varphi_{R}(\Delta)
$$

so that

$$
\tilde{Z}_{n}(\xi)=\sum_{R} d_{R}^{2} e^{\xi_{R}}=Z_{n}(\beta)
$$

Then the WDVV equations are trivially satisfied and, since this is no more than a linear (and non-degenerate) change of beta-variables, the original WDVV equations for $Z_{n}(\beta)$ are also true.

It is also evident that if one inserts into the sum (72) an arbitrary product of characters $\chi_{R}\left(t_{k}^{(1)}\right) \ldots \chi_{R}\left(t_{k}^{(m)}\right)$, it does not spoil the argument and, hence, the WDVV equations are still satisfied. 


\subsection{The *-product case}

In this case one has to consider the WDVV equations with infinite matrices: there is no any simple finite truncation for them. For instance, the simplest WDVV equation $\check{C}_{2} \check{C}_{3}=\check{C}_{3} \check{C}_{2}(23)$ is

$\frac{<112><123>}{<11>}+\frac{<122><223>}{<22>}+\frac{<123><233>}{<33>}=\frac{<113><122>}{<11>}+\frac{<123><222>}{<22>}+\frac{<133><223>}{<33>}$

and it does not hold with

$$
\begin{array}{r}
<123>=<[1] *[2] *[3]>=0, \quad<113>=0, \\
<122>=3 / 2, \quad<223>=1, \quad<133>=4 / 3, \\
<11>=1, \quad<22>=1 / 2, \quad<33>=1 / 3
\end{array}
$$

Indeed, vanishing entries in the first line reduce the equation just to

$$
\frac{<122><223>}{<22>}=\frac{<133><223>}{<33>}
$$

which is not true.

What is the reason? In fact, $\check{C}_{2} \check{C}_{3}=\check{C}_{3} \check{C}_{2}$ holds in the following way:

$$
\begin{array}{r}
<(2 * 2) * 3>=C_{22}^{3}<3 * 3>=C_{22}^{3} C_{33}^{111}<111>=3 \cdot 2 \cdot \frac{1}{6}, \\
<2 *(2 * 3)>=C_{23}^{[21]}<2 *[21]>=C_{32}^{[21]} C_{[21], 2}^{111}<111>=3 \cdot 2 \cdot \frac{1}{6}
\end{array}
$$

i.e. as ${ }_{2}\left(\check{C}_{2} \check{C}_{3}=\check{C}_{3} \check{C}_{2}\right)^{[111]}$ :

$$
C_{22}^{3} C_{33}^{111}=C_{32}^{[21]} C_{[21], 2}^{111}
$$

However, while

$$
C_{22}^{3} C_{33}^{111}=\frac{<2 * 2 * 3>}{<3 * 3>} \frac{<* 3 * 111>}{<111 * 111>}=\frac{<3 * 2 * 21>}{<21 * 21>} \frac{<* 21 * 111>}{<111 * 111>}=C_{32}^{[21]} C_{[21], 2}^{111}
$$

the symbolical expression neglecting the number of units does not hold:

$$
C_{22}^{3} C_{33}^{1} \stackrel{?}{=} \frac{<223>}{<33>} \frac{<133>}{<11>} \neq \frac{<223>}{<22>} \frac{<122>}{<11>} \stackrel{?}{=} C_{32}^{2} C_{22}^{1}
$$

Thus, reduction does not take place in the 1-sector.

Thus, one has to deal with infinite matrices. Though the WDVV equations still have to be satisfied, checking them directly is a non-trivial problem. This is easier to do in the simpler case of the $\left[1^{p}\right]$-subring case, which we discuss in the next two sections.

\section{Sub-ring of $\left[1^{p}\right]$ operators and its action on entire algebra}

In this section we discuss multiplication by the $\left[1^{p}\right]$ operators, which form a closed sub-algebra of the entire algebra. Moreover, in this case, it is possible to write down general formulas.

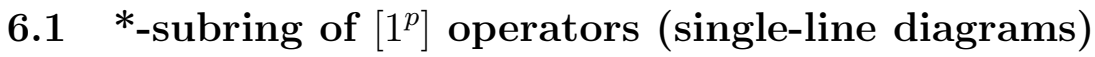

The multiplication of $\left[1^{p}\right]$ operators is given by the formula

$$
\left[1^{p}\right] *\left[1^{q}\right]=\sum_{i=\max (0, p-q)}^{p} \frac{(q+i) !}{i !(p-i) !(q-p+i) !}\left[1^{q+i}\right]=\sum_{s=\max (p, q)}^{p+q} \frac{s !}{(s-p) !(s-q) !(p+q-s) !}\left[1^{s}\right]
$$

Introduce $I(x)=\sum_{p} x^{p}\left[1^{p}\right]$. Then it follows that

$$
\begin{array}{r}
I(x) * I(y)=I(x+y+x y), \\
I(x) * I(y) * I(z)=I(x+y+x y) * I(z)=I(x+y+z+x y+y z+z x+x y z), \\
\ldots, \\
*_{i=1}^{m} I\left(x_{i}\right)=I\left(\sum_{i} x_{i}+\sum_{i<j} x_{i} x_{j}+\sum_{i<j<k} x_{i} x_{j} x_{k}+\ldots+\prod_{i=1}^{m} x_{i}\right)=I\left(-1+\prod_{i}\left(x_{i}+1\right)\right)
\end{array}
$$




\subsection{Action of $\left[1^{p}\right]$ operators on the entire algebra}

The multiplication of any operator by $\left[1^{p}\right]$ operators is described with the following formulas. Let $\Delta=\left[\tilde{\Delta}, 1^{r}\right]$, with $1 \notin \tilde{\Delta}$. Let first $p \leq m=|\Delta|=|\tilde{\Delta}|+r$ :

$$
\left.\begin{array}{r}
{\left[1^{p}\right] *[\Delta]=\sum_{i=0}^{p}\left\{1^{p}, \Delta\right\}_{m+i},} \\
\left\{1^{p}, \Delta\right\}_{m}=\rho_{m-p}\left[1^{p}\right] \circ \Delta=\frac{m !}{p !(m-p) !}\left[1^{m}\right] \circ \Delta=C_{m}^{p} \Delta, \\
\left\{1^{p}, \Delta\right\}_{m+1}=\rho_{m-p+1}\left[1^{p}\right] \circ \rho_{1}[\Delta]-\rho_{1}\left(\left\{1^{p}, \Delta\right\}_{m}\right)= \\
=C_{m+1}^{p}\left[1^{m+1}\right] \circ(r+1)[\Delta, 1]-C_{m}^{p} \cdot(r+1)[\Delta, 1]=(r+1) C_{m}^{p-1}[\Delta, 1], \\
\left\{1^{p}, \Delta\right\}_{m+2}=\rho_{m-p+2}\left[1^{p}\right] \circ \rho_{2}[\Delta]-\rho_{2}\left(\left\{1^{p}, \Delta\right\}_{m}\right)-\rho_{1}\left(\left\{1^{p}, \Delta\right\} m+1\right)= \\
=C_{m+2}^{p}\left[1^{m+2}\right] \circ \frac{(r+1)(r+2)}{2}[\Delta, 1,1]-\frac{(r+1)(r+2)}{2} C_{m}^{p}[\Delta, 1,1]-(r+2) \cdot(r+1) C_{m}^{p-1}[\Delta, 1,1]= \\
=\frac{(r+1)(r+2)}{2}\left(C_{m+2}^{p}-C_{m}^{p}-2 C_{m}^{p-1}\right)[\Delta, 1,1]=C_{r+2}^{2} C_{m}^{p-2}[\Delta, 1,1], \\
\ldots \\
{\left[1^{p}\right] * \Delta=C_{m}^{p} \Delta+(r+1) C_{m}^{p-1}[\Delta, 1]+C_{r+2}^{2} C_{m}^{p-2}[\Delta, 1,1]+\ldots=\sum_{i=0}^{p} C_{r+i}^{i} C_{m}^{p-i}\left[\Delta, 1^{i}\right]}
\end{array}\right]
$$

In this form the formula holds also for $p>m$, just the sum actually goes from $i=\max (0, p-m)$.

Eq.(84) can be rewritten also as

$$
\left[1^{p}\right] *\left[\tilde{\Delta}, 1^{r}\right]=\sum_{i=0}^{p} C_{r+i}^{i} C_{|\tilde{\Delta}|+r}^{p-i}\left[\tilde{\Delta}, 1^{r+i}\right]
$$

and, further,

$$
I(x) *\left[\tilde{\Delta}, 1^{r}\right]=\sum_{p, i} x^{p} C_{|\Delta|}^{p-i} C_{r+i}^{i}\left[\tilde{\Delta}, 1^{i+r}\right]=(1+x)^{|\Delta|} \sum_{i} x^{i} C_{r+i}^{i}\left[\tilde{\Delta}, 1^{i+r}\right]
$$

If we introduce now a new generating function $I_{\tilde{\Delta}}(x)=\sum_{p} x^{p}\left[\tilde{\Delta}, 1^{p}\right]$, then

$$
I(x) * I_{\tilde{\Delta}}(y)=(1+x)^{|\tilde{\Delta}|} \sum_{i, r} C_{r+i}^{i} x^{i}(1+x)^{r} y^{r}\left[1^{r+i}\right]=(1+x)^{|\tilde{\Delta}|} I_{\tilde{\Delta}}(x+y+x y)
$$

This formula describes the action of the $\left[1^{p}\right]$-subring on the entire algebra of cut-and-join operators.

\section{3 o-correlators in the $\left[1^{p}\right]$ subring}

The o-multiplication is much simpler and in the $\left[1^{p}\right]$ sector can be written by the two generating functions. The first one is

$$
\sum_{n=0}^{\infty} q^{n}\left\langle\left[1^{n}\right] \circ\left[1^{n}\right]\right\rangle t_{1}^{n} \bar{t}_{1}^{n}=\sum_{n=0}^{\infty} q^{n}\left\langle\left[1^{n}\right]\right\rangle t_{1}^{n} \bar{t}_{1}^{n}=\sum_{n=0}^{\infty} \frac{q^{n} t_{1}^{n} \bar{t}_{1}^{n}}{n !}=\exp \left(q t_{1} \bar{t}_{1}\right)
$$

Similarly,

$$
\sum_{n=0}^{\infty} q^{n}\left\langle\left[1^{n}\right]^{\circ m}\right\rangle\left(t_{1}^{(1)} \ldots t_{1}^{(m)}\right)^{n}=\exp \left(q t_{1}^{(1)} \ldots t_{1}^{(m)}\right)
$$

The second generating function is

$$
\sum_{n=0}^{\infty} q^{n}\left\langle\exp _{\circ}\left(\beta_{1^{n}}\left[1^{n}\right]\right)\right\rangle=\sum_{n=0}^{\infty} q^{n}\left\langle\delta_{n, 0}+\beta_{1^{n}}\left[1^{n}\right]+\frac{\beta_{1^{n}}^{2}}{2 !}\left[1^{n}\right] \circ\left[1^{n}\right]+\ldots\right\rangle=\sum_{n=0}^{\infty} \frac{q^{n} e^{\beta_{1^{n}}}}{n !}
$$

While each item in the sum is an average of a o-exponential, the sum over $n$ is not, and one can not expect that such partition functions satisfy the WDVV equations. 
One can also consider a simplified version of (90), with all $\beta^{1^{n}}$ equal: $\beta_{1^{n}}=\beta_{1}$. Then (90) turns into

$$
\sum_{n=0}^{\infty} q^{n}\left\langle\exp _{\circ}\left(\beta_{1}\left[1^{n}\right]\right)\right\rangle=\sum_{n=0}^{\infty} q^{n}\left\langle\exp _{\circ}\left(\beta_{1} \rho_{n-1}[1]\right)\right\rangle=e^{\beta_{1}+q}
$$

The standard Hurwitz partition function is direct generalization of this formula.

\subsection{Connecting two multiplications}

Formula (43) connecting the two multiplications can be further specified for the $\left[1^{p}\right]$ subring. Define in this case one more generating function

$$
\sum_{p} x^{p} J_{\left[1^{p}\right]}(u)=\sum_{p, m \geq 0} u^{p+m} x^{p} \rho_{m}\left(\left[1^{p}\right]\right)=\sum_{m, p \geq 0} \frac{(m+p) !}{m ! p !} u^{p+m} x^{p}\left[1^{p+m}\right]
$$

Then, in terms of $I(u)=\sum_{m} u^{m}\left[1^{m}\right]$, one has: $J_{\left[1^{p}\right]}(u)=u^{p} \partial_{u}^{p} I(u) / p$ ! and one can easily relate (43) to $I(x) * I(y)=I(x+y+x y)$, so that

$$
\oint J_{I(x)}(u) \circ J_{I(y)}\left(\frac{v}{u}\right) \frac{d u}{u}=J_{I(x+y+x y)}(v)
$$

Note that in the generic case it would be interesting to consider a generating function with the full set of time variable $\left\{p_{k}\right\}, J(u \mid p)=\sum_{\Delta} J_{\Delta}(u) p_{\Delta}$, so that

$$
\oint J(u \mid p) \circ J\left(\frac{v}{u} \mid \bar{p}\right) \frac{d u}{u}=\sum_{\Delta_{1}, \Delta_{2}} J_{\Delta_{1} * \Delta_{2}}(v)
$$

and realize if there is an interesting expression for the r.h.s.

\section{Generalized Hurwitz partition function for the $\left[1^{p}\right]$ subring}

\section{$7.1 *$-correlators}

Averaging converts $I(x)$ into the exponential:

$$
\begin{array}{r}
\langle I(x)\rangle=\sum_{p} \frac{x^{p}}{p !}=e^{x}, \\
\left\langle *_{i} I\left(x_{i}\right)\right\rangle=\exp \left(-1+\prod_{i}\left(x_{i}+1\right)\right)
\end{array}
$$

or

$$
1+\log \left\langle *_{i} I\left(x_{i}\right)\right\rangle=\prod_{i}\left(1+x_{i}\right)
$$

In particular,

$$
\sum_{k, l=0}^{\infty}\left\langle\left[1^{k}\right] *\left[1^{l}\right]\right\rangle t_{1}^{k} \bar{t}_{1}^{l}=<I\left(t_{1}+\bar{t}_{1}+t_{1} \bar{t}_{1}\right)>=\exp \left(t_{1}+\bar{t}_{1}+t_{1} \bar{t}_{1}\right)
$$

Similarly

$$
\sum_{k_{1}, \ldots, k_{m}=0}^{\infty}\left\langle\left[1^{k_{1}}\right] * \ldots *\left[1^{k_{m}}\right]\right\rangle\left(t_{1}^{(1)}\right)^{k_{1}} \ldots\left(t_{1}^{(m)}\right)^{k_{m}}=\exp \left(-1+\prod_{i=1}^{m}\left(\left(1+t_{m}^{(i)}\right)\right)\right.
$$




\subsection{Correlators of $*$-exponentials}

It is convenient to introduce a grading of the diagram by its rescaling with a formal parameter $q:\left[1^{p}\right] \rightarrow q^{p}\left[1^{p}\right]$. The rescaled diagrams (operators $q^{p} \hat{W}\left[1^{p}\right]$ ) also form a *-ring, but with rescaled structure constants. In terms of the generating function $I_{q}(x)=\sum_{p} x^{p} q^{p}\left[1^{p}\right]=I(q x)$ one has $I_{q}(x) * I_{q}(y)=I(q x) * I(q y)=I_{q}(x+y+q x y)$. Note that for the o-multiplication $[1]^{p} \circ\left[1^{p}\right]=\left[1^{p}\right]$ and logarithm of the average $\log \left\langle\sum_{p, q} x^{p} y^{q}[1]^{p} \circ\left[1^{q}\right] \delta_{p, q}\right\rangle=x y$ is obtained from $\frac{1}{q} \log <I_{q}(x) * I_{q}(y)>=\frac{x+y}{q}+x y$ in the limit of $q \rightarrow \infty$.

In order to check the associativity, one has to define the partition function

$$
Z_{*}\{\beta \mid q\}=\left\langle\exp _{*}\left(\sum_{p} \beta_{\left[1^{p}\right]} q^{p}\left[1^{p}\right]\right)\right\rangle=1+\sum_{p} \frac{1}{p !} q^{p} \beta_{p}+\frac{1}{2 !} \sum_{p_{1}, p_{2}} \beta_{p_{1}} \beta_{p_{2}} \oint \frac{d x}{x^{p_{1}+1}} \oint \frac{d y}{y^{p_{2}+1}} e^{x+y+q x y}+\ldots
$$

and check if its third derivatives w.r.t. $\beta$ 's satisfy the WDVV equations.

This quantity can be studied using the technique developed above. For checks of the equation, one can use the perturbative expansion of $Z_{*}\{\beta \mid q\}$ into the power series in $q$. Let us see how this works in the leading order.

\subsection{WDVV equations for the $\left[1^{p}\right]$ subring}

Despite even for this subring the partition function depends on infinitely many variables and, hence, the matrices of the third derivatives

$$
\left(\hat{C}_{i}\right)_{j k} \equiv \hat{C}_{i j k}=C_{i j k}=\frac{\partial^{3} Z_{*}\{\beta \mid q\}}{\partial \beta_{i} \partial \beta_{j} \partial \beta_{k}}
$$

is infinite-dimensional, the associativity equations can be explicitly checked.

In the leading order approximation one has to check the associativity of the non-deformed structure constants, i.e. (100) calculated at all $\beta_{k}=0$. The generating functions for the structure constants $A_{p q}^{s}$ of the subring,

$$
\left[1^{p}\right] *\left[1^{q}\right]=\sum_{s=\max (p, q)}^{p+q} A_{p q}^{s}\left[1^{s}\right]
$$

are given by

$$
A^{s}(x, y)=\sum_{p, q} A_{p q}^{s} x^{p} y^{q}=(x+y+x y)^{s}
$$

or

$$
A(x, y ; u)=\sum_{p, q, s} A_{p q}^{s} \frac{x^{p} y^{q}}{u^{s+1}}=\frac{1}{u-(x+y+x y)}
$$

The associativity is guaranteed by the symmetricity of

$$
\sum_{p, q, t} x^{p} y^{q} z^{t} \sum_{s} A_{p q}^{s} A_{s t}^{r}=\oint A(x, y ; u) A^{r}(u, z) d u=(x+y+z+x y+y z+x z+x y z)^{r}
$$

w.r.t. $x \leftrightarrow z$ and $y \leftrightarrow z$.

Associativity condition (104) should be complemented by

$$
\begin{aligned}
<\left[1^{p}\right] *\left[1^{q}\right] *\left[1^{r}\right]> & =\sum_{s} A_{p q}^{s}<\left[1^{s}\right] *\left[1^{r}\right]>, \\
e^{x+y+z+x y+y z+x z+x y z} & =\oint A(x, y ; u) e^{u+z+u z} d u
\end{aligned}
$$

which proves the WDVV equations in the leading order.

Further, one can switch on perturbations and check the WDVV equations explicitly in higher orders. We certainly know that they are correct basing on the general grounds, however, the procedure described here allows one to check this iteratively. 


\section{Acknowledgements}

Our work is partly supported by Russian Federal Nuclear Energy Agency under contract H.4e.45.90.11.1059, by Ministry of Education and Science of the Russian Federation under contract 02.740.11.0608, by Russian government grant 11.G34.31.005, by RFBR grants 10-02-00509 (A.Mir.), 10-02-00499 (A.Mor.) and 11-0100289 (S.N.), by joint grants 11-02-90453-Ukr, 09-02-93105-CNRSL, 09-02-91005-ANF, 10-02-92109-Yaf-a, 11 01-92612-Royal Society and by grant NSh 8462.2010.1.

\section{References}

[1] A.Hurwitz, Math.Ann. 39 (1891) 1-61; Math.Ann. bf 55 (1902) 51-60

[2] G.Frobenius, Sitzberg. Koniglich Preuss. Akad.Wiss. Berlin(1896) 985-1021

[3] R.Dijkgraaf, In: The moduli spaces of curves, Progress in Math., 129 (1995), 149-163, Brikhäuser

[4] R.Vakil, Enumerative geometry of curves via degeneration methods, Harvard Ph.D. thesis (1997)

[5] I.Goulden and D.Jackson, Proc.Amer.Math.Soc. 125 (1997) 51-60, math/9903094

[6] S.Lando and D.Zvonkine, Funk.Anal.Appl. 333 (1999) 178-188; math.AG/0303218

[7] S.Natanzon and V.Turaev, Topology, 38 (1999) 889-914

[8] Goulden D., Jackson D.M., Vainshtein A., Ann. of Comb. 4(2000), 27-46, Brikhäuser

[9] A.Okounkov, Math.Res.Lett. 7 (2000) 447-453

[10] A.Givental, math/0108100

[11] T.Ekedahl, S.Lando, M.Shapiro, A.Vainshtein, Invent.Math.146(2001),297-327

[12] S.Lando, Russ.Math.Surv., 57 (2002) 463-533

[13] A.Alexeevski and S.Natanzon, Selecta Math., New ser. 12:3 (2006) 307-377, math.GT/0202164

[14] S.Natanzon, Russian Math.Survey 61:4 (2006) 185-186; arXiv:0804.0242

A.Alexeevski and S.Natanzon, Amer.Math.Soc.Transl. 224 (2) (2008) 1-25; Izvestia RAN, 12:4 (2008) 3-24

[15] J.Zhou, math.AG/0308024

[16] A.Okounkov and R.Pandharipande, Ann. of Math. 163 (2006) 517, math.AG/0204305

[17] T.Graber and R.Vakil, Compositio Math., 135 (2003) 25-36

[18] M.Kazarian and S.Lando, math.AG/0410388; math/0601760

[19] M.Kazarian, arXiv:0809.3263

[20] S.Lando, In: Applications of Group Theory to Combinatorics, Koolen, Kwak and Xu, Eds. Taylor \& Francis Group, London, 2008, 109-132

[21] V.Bouchard and M.Marino, In: From Hodge Theory to Integrability and tQFT: $t t^{*}$-geometry, Proceedings of Symposia in Pure Mathematics, AMS (2008), arXiv:0709.1458

[22] A.Mironov and A.Morozov, JHEP 0902 (2009) 024, arXiv:0807.2843

[23] A.Morozov and Sh.Shakirov, JHEP, 0904 (2009) 064, arXiv: 0902.2627; Mod.Phys.Lett. A24 (2009) 26592666, arXiv:0906.2573

[24] G.Borot, B.Eynard, M.Mulase and B.Safnuk, arXiv:0906.1206

[25] A.Alexandrov, arXiv:1005.5715

[26] A.Mironov, A.Morozov and S.Natanzon, Theor.Math.Phys. 166 (2011) 1-22, arXiv:0904.4227; arXiv:1012.0433 
[27] A.Alexandrov, A.Mironov, A.Morozov and S.Natanzon, arXiv:1103.4100

[28] D.E.Littlewood, The theory of group characters and matrix representations of groups, Oxford, 1958 I.G.Macdonald, Symmetric functions and Hall polynomials, Oxford Science Publications, 1995 W.Fulton, Young tableaux: with applications to representation theory and geometry, London Mathematical Society, 1997

[29] M.Hamermesh, Group theory and its application to physical problems, 1989

[30] V.Ivanov, S.Kerov, Journal of Mathematical Sciences (Kluwer) 107 (2001) no.5 4212-4230, arXiv:math/0302203

[31] A.Okounkov and G.Olshanski, St.Petersburg Math.J. 9 (1998) no. 2, arXiv:q-alg/9605042

[32] A.Alexandrov et al., to appear

[33] A.Marshakov, A.Mironov and A.Morozov, Phys.Lett., B389 (1996) 43, hep-th/9607109

[34] I.Krichever, hep-th/9205110; Comm.Math.Phys. 143 (1992) 415; A.Gorsky, A.Marshakov, A.Mironov and A.Morozov, Nucl.Phys., B527 (1998) 690-716; hep-th/9802007; A.Boyarsky, A.Marshakov, O.Ruchayskiy, P.Wiegmann and A.Zabrodin, Phys.Lett. B515 (2001) 483-492, hep-th/0105260

[35] K.Takasaki, arXiv:1012.5554

[36] E.Witten, Surv.Diff.Geom., 1 (1991) 243

R.Dijkgraaf, E.Verlinde and H.Verlinde, Nucl.Phys., B352 (1991) 59;

I.Krichever, Comm.Pure Appl.Math., 47 (1994) 437;

B.Dubrovin, Nucl.Phys., B379 (1992) 627; hep-th/9407018

[37] S.Natanzon and V.Turaev, Comm.Math.Phys. 196 (1998) 399-410

[38] A.Marshakov, A.Mironov and A.Morozov, Int.J.Mod.Phys., A15 (2000) 1157-1206, hep-th/9701123; Mod.Phys.Lett., A12 (1997) 773, hep-th/9701014;

A.Mironov and A.Morozov, Phys.Lett., B424 (1998) 48-52, hep-th/9712177;

A.Mironov, in: H.W.Braden and I.M.Krichever (Eds.), Integrability: The Seiberg-Witten and Whitham Equations, Gordon and Beach, 2000, arXiv:hep-th/9903088;

A.Veselov, Phys.Lett., A261 (1999) 297-302, hep-th/9902142;

Feigin and A.P.Veselov, arXiv:0710.5729;

O.Lechtenfeld, K.Schwerdtfeger and J.Thürigen, SIGMA 7 (2011) 023, arXiv:1011.2207

[39] S.Kharchev, A.Marshakov, A.Mironov and A.Morozov, Int. J. Mod. Phys. A10 (1995) 2015, hepth/9312210 


\section{Appendices}

\section{Appendix I. Multiplications of compositions.}

We illustrate the use of formulae of s.3 with the following example: $\left[2,1^{r}\right] \circ\left[2,1^{r}\right]$. Here $\left[2,1^{r}\right]=(12)+\ldots$ is a sum of $\left\|2,1^{r}\right\|=C_{r+2}^{2}=\frac{(r+1)(r+2)}{2}$ permutations. In the o-product each of them act on the permutations in another $\left[2,1^{r}\right]$, and the action of, say, (12) by the rules (32) is different on (12), (13) and (34). Then, on (14) it is the same as on (13) and on (56) - the same as on (34) - the same in the sense that it produces an element in the same conjugation class. According to (32),

$$
\begin{array}{r}
(12) \circ(12)=() \in\left[1^{r+2}\right], \\
(12) \circ(13)=(123) \in\left[3,1^{r-1}\right], \\
(12) \circ(34)=(12)(34) \in\left[22,1^{r-2}\right]
\end{array}
$$

It remains to calculate the multiplicities:

$$
\begin{array}{r}
\left\|2,1^{r-1}\right\| \cdot 1 /\left\|1^{r+2}\right\|=\left\|2,1^{r}\right\|=C_{r+2}^{2}=\frac{(r+1)(r+2)}{2}, \\
\left\|2,1^{r-1}\right\| \cdot 2 r /\left\|3,1^{r-1}\right\|=C_{r+2}^{2} \cdot 2 r / 2 C_{r+2}^{3}=3, \\
\left\|2,1^{r-1}\right\| \cdot C_{r}^{2} /\left\|22,1^{r-2}\right\|=C_{r+2}^{2} \cdot C_{r}^{2} /\left(C_{r+2}^{2} C_{r}^{2} / 2\right)=2
\end{array}
$$

Here $1,2 r$ and $C_{r}^{2}$ are the numbers of permutations of the types (12), $(1 k)$ or $(2 k)$ with $k \neq 1,2$ and $(k l)$ with $k, l \neq 1,2$ in the conjugation class $\left[2,1^{r}\right]$. Of course, $1+2 r+C_{r}^{2}=\left\|2,1^{r}\right\|=C_{r+2}^{2}$. Thus we obtain:

$$
\left[2,1^{r}\right] \circ\left[2,1^{r}\right]=\frac{(r+1)(r+2)}{2}\left[1^{r+2}\right]+3\left[3,1^{r-1}\right]+2\left[22,1^{r-2}\right]
$$

Similarly,

$$
\left[2,1^{r}\right] \circ\left[3,1^{r-1}\right]=2 r\left[2,1^{r}\right]+\left[32,1^{r-3}\right]+4\left[4,1^{r-2}\right]
$$

and so on.

- Similarly, for $\left[3,1^{m}\right]$ :

$$
\begin{array}{r}
(123) \circ(123) K=(132) K, \\
(123) \circ(132) K=K, \\
(123) \circ(12 C) K=(1 C)(23) K, \\
(123) \circ(13 C) K=(12 C) K, \\
(123) \circ(1 C) K=(123 C) K, \\
(123) \circ(C) K=(123) C K, \\
(123) \circ\left(12 C_{1}\right)\left(3 C_{2}\right) K=\left(12 C_{1} 3 C_{2}\right) K \\
(123) \circ\left(13 C_{1}\right)\left(2 C_{2}\right) K=\left(13 C_{1} 2 C_{2}\right) K \\
(123) \circ\left(1 C_{1}\right)\left(2 C_{2}\right)\left(3 C_{3}\right) K=\left(1 C_{1} 2 C_{2} 3 C_{3}\right) K
\end{array}
$$

Therefore

$$
[3111] \circ[3111]=40\left[1^{6}\right]+8[2211]+10[3111]+2[33]+5[51]
$$

and in general

$$
\begin{array}{r}
{\left[3,1^{r}\right] \circ\left[3,1^{r}\right]=\frac{2 C_{r+3}^{3}}{2 C_{r+3}^{3}}\left[3,1^{r}\right]+\frac{2 C_{r+3}^{3}}{1}\left[1^{r+3}\right]+\frac{2 C_{r+3}^{3} \cdot 3 r}{C_{r+3}^{2} C_{r+1}^{2} / 2}\left[22,1^{r-1}\right]+} \\
+\frac{2 C_{r+3}^{3} \cdot 3 r}{2 C_{r+3}^{3}}\left[3,1^{r}\right]+\frac{2 C_{r+3}^{3} \cdot 6 C_{r}^{2}}{24 C_{r+3}^{5}}\left[5,1^{r-2}\right]+\frac{2 C_{r+3}^{3} \cdot 2 C_{r}^{3}}{2 C_{r+3}^{3} \cdot 2 C_{r}^{3} / 2}\left[33,1^{r-3}\right]= \\
=\frac{(r+3)(r+2)(r+1)}{3}\left[1^{r+3}\right]+8\left[22,1^{r-1}\right]+(3 r+1)\left[3,1^{r}\right]+8\left[33,1^{r-3}\right]+5\left[5,1^{r-2}\right]
\end{array}
$$


A check of multiplicities in this formula:

$$
1+1+2 \cdot 3 r+6 C_{r}^{2}+2 C_{r}^{3}=2 C_{r+3}^{3}
$$

- The same formulas (110) can be used to handle more general o-products involving the triple cycles. In particular,

$$
\begin{array}{r}
{[3111] \circ[33]=2[311]+2[33]+12[6],} \\
{\left[3,1^{4}\right] \circ[331]=8\left[3,1^{4}\right]+2[331]+8[43]+18[61]}
\end{array}
$$

and in general

$$
\begin{array}{r}
{\left[3,1^{r+3}\right] \circ\left[33,1^{r}\right]=\frac{(r+1)(r+2)(r+3)}{3}\left[3,1^{r+3}\right]+2\left[33,1^{r}\right]+3\left[333,1^{r-3}\right]+} \\
+8\left[43,1^{r-1}\right]+5\left[531^{r-2}\right]+6(r+2)\left[61^{r}\right]
\end{array}
$$

\section{Appendix II. Multiplication tables}

\subsection{Group $S(2)$}

$$
\|11\|=1, \quad\|2\|=1
$$

\begin{tabular}{|c|c|}
\hline$[11]$ & {$[2]$} \\
\hline$[2]$ & {$[11]$} \\
\hline
\end{tabular}

\subsection{Group $S(3)$}

$$
\begin{array}{ll}
{[111]=(),} & \|111\|=1 \\
{[21]=(12)+(13)+(23),} & \|21\|=3 \\
{[3]=(123)+(132),} & \|3\|=2
\end{array}
$$

\begin{tabular}{|c|c|c|}
\hline$[111]$ & {$[21]$} & {$[3]$} \\
\hline$[21]$ & $3[111]+3[3]$ & $2[21]$ \\
\hline$[3]$ & $2[21]$ & $2[111]+[3]$ \\
\hline
\end{tabular}

\subsection{Group $S(4)$}

$$
\begin{array}{ll}
{[1111]=(),} & \|1111\|=1 \\
{[211]=(12)+(13)+(14)+(23)+(24)+(34),} & \|211\|=6 \\
{[22]=(12)(34)+(13)(24)+(14)(23),} & \|22\|=3 \\
{[31]=(123)+(132)+(124)+(142)+(134)+(143)+(234)+(243),} & \|31\|=8 \\
{[4]=(1234)+(1243)+(1324)+(1342)+(1423)+(1432),} & \|4\|=6
\end{array}
$$

\begin{tabular}{|c|c|c|c|c|}
\hline$[1111]$ & {$[211]$} & {$[22]$} & {$[31]$} & {$[4]$} \\
\hline$[211]$ & $6[1111]+2[22]+3[31]$ & {$[211]+2[4]$} & $4[211]+4[4]$ & $4[22]+3[31]$ \\
\hline$[22]$ & {$[211]+2[4]$} & $3[1111]+2[22]$ & $3[31]$ & $2[211]+[4]$ \\
\hline$[31]$ & $4[211]+4[4]$ & $3[31]$ & $8[1111]+8[22]+4[31]$ & $4[211]+4[4]$ \\
\hline$[4]$ & $4[22]+3[31]$ & $2[211]+[4]$ & $4[211]+4[4]$ & $6[1111]+2[22]+3[31]$ \\
\hline
\end{tabular}


7.7 Group $S(5)$

$$
\begin{array}{ll}
{[11111]=(),} & \|11111\|=1 \\
{[2111]=(12)+\ldots,} & \|2111\|=C_{5}^{2}=10 \\
{[221]=(12)(34)+\ldots,} & \|221\|=\frac{1}{2} C_{5}^{2} C_{3}^{2}=15 \\
{[311]=(123)+(132)+\ldots,} & \|311\|=2 C_{5}^{3}=20 \\
{[32]=(123)(45)+\ldots,} & \|32\|=\|311\|=20 \\
{[41]=(1234)+\ldots,} & \|41\|=3 ! C_{5}^{1}=30 \\
{[5]=(12345)+\ldots} & \|5\|=4 !=24 \\
\sum_{\Delta:|\Delta|=5}\|\Delta\|=5 !=120
\end{array}
$$

\begin{tabular}{|c|c|c|c|c|c|c|}
\hline$[11111]$ & {$[2111]$} & {$[221]$} & {$[311]$} & {$[32]$} & {$[41]$} & {$[5]$} \\
\hline$[2111]$ & $10[11111]+2[221]+3[311]$ & $3[2111]+3[32]+2[41]$ & $6[2111]+[32]+4[41]$ & $4[221]+[311]+5[5]$ & $9[311]+5[5]$ & $8[41]$ \\
\hline$[221]$ & $3[2111]+3[32]+2[41]$ & & & & & \\
\hline$[311]$ & $6[2111]+[32]+4[41]$ & & $\begin{array}{c}20[11111]+8[221]+ \\
+7[311]+5[5]\end{array}$ & & & \\
\hline$[32]$ & $4[221]+[311]+5[5]$ & & & & & \\
\hline$[41]$ & $9[311]+5[5]$ & & & & & \\
\hline$[5]$ & $8[41]$ & & & & & \\
\hline
\end{tabular}

\section{Appendix III. Structure constants of $*$-multiplication}

\subsection{Level $(1,1)$}

When it does not cause confusion we omit square brackets in the notation of particular Young diagrams, to simplify the formulas.

$$
\begin{array}{r}
{[1] *[1]=\{1,1\}_{1}+\{1,1\}_{2},} \\
\{1,1\}_{1}=[1] \circ[1]=[1], \\
\{1,1\}_{2}=\rho_{1}[1] \circ \rho_{1}[1]-\rho_{1}([1] \circ[1])=\rho_{1}[1] \circ \rho_{1}[1]-\rho_{1}([1])=2[11] \circ 2[11]-2 \circ[11]=2[11]
\end{array}
$$

Thus

$$
[1] *[1]=\underline{[1]}+2[11]
$$

Here and below we underline the terms in the product, which belong to the o-product: they exist only when $\left|\Delta_{1}\right|=\left|\Delta_{2}\right|$ and are contained in the first term $\left\{\Delta_{1}, \Delta_{2}\right\}_{\left|\Delta_{1}\right|=\left|\Delta_{2}\right|}=\Delta_{1} \circ \Delta_{2}$ with $n=\left|\Delta_{1}\right|=\left|\Delta_{2}\right|$ in the sum in (37).

\subsection{Level $(1,2)$}

$$
\begin{array}{r}
{[1] *[11]=\{1,11\}_{2}+\{1,11\}_{3},} \\
\{1,11\}_{2}=\rho_{1}[1] \circ[11]=2[11] \circ[11]=2[11], \\
\{1,11\}_{3}=\rho_{2}[1] \circ \rho_{1}[11]-\rho_{1}\left(\{1,11\}_{2}\right)=3[111] \circ 3[111]-2 \cdot 3[111]=3[111], \\
{[1] *[11]=2[11]+3[111]}
\end{array}
$$

and

$$
\begin{array}{r}
{[1] *[2]=\{1,2\}_{2}+\{1,2\}_{3},} \\
\{1,2\}_{2}=\rho_{1}[1] \circ[2]=2[11] \circ[2]=2[2], \\
\{1,2\}_{3}=\rho_{2}[1] \circ \rho_{1}[2]-\rho_{1}\left(\{1,2\}_{2}\right)=3[111] \circ[21]-2[21]=[21], \\
{[1] *[2]=2[2]+[21]}
\end{array}
$$




\subsection{Level $(1,3)$}

$$
\begin{gathered}
{[1] *[111]=\{1,111\}_{3}+\{1,111\}_{4},} \\
\{1,111\}_{3}=\rho_{2}[1] \circ[111]=3[111] \circ[111]=3[111], \\
\{1,111\}_{4}=\rho_{3}[1] \circ \rho_{1}[111]-\rho_{1}\left(\{1,111\}_{3}\right)=4[1111 \circ 4[1111]-4 \cdot 3[1111]=4[1111], \\
{[1] *[111]=3[111]+4[1111]} \\
\{1] *[21]=\{1,21\}_{3}+\{1,21\}_{4}, \\
\{1,21\}_{3}=\rho_{2}[1] \circ[21]=3[111] \circ[21]=3[21], \\
\{1,21\}_{4}=\rho_{3}[1] \circ \rho_{1}[21]-\rho_{1}\left(\{1,21\}_{3}\right)=4[1111] \circ 2[211]-2 \cdot 3[211]=2[211], \\
{[1] *[21]=3[21]+2[211]} \\
\{1] *[3]=\{1,3\}\}_{3}+\{1,3\}_{4}, \\
\{1,3\}_{3}=\rho_{2}[1] \circ[3]=3[111] \circ[3]=3[3], \\
{[1] *[3]=3[3]+[31]}
\end{gathered}
$$

\subsection{Level $(2,2)$}

$$
\begin{aligned}
& {[11] *[11]=\{11,11\}_{2}+\{11,11\}_{3}+\{11,11\}_{4},} \\
& \{11,11\}_{2}=[11] \circ[11]=[11], \\
& \{11,11\}_{3}=\rho_{1}[11] \circ \rho_{1}[11]-\rho_{1}\left(\{11,11\}_{2}\right)=3[111] \circ 3[111]-3[111]=6[111], \\
& \{11,11\}_{4}=\rho_{2}[11] \circ \rho_{2}[11]-\rho_{2}\left(\{11,11\}_{2}\right)-\rho_{1}\left(\{11,11\}_{3}\right)=6[1111] \circ 6[1111]-6[1111]-4 \cdot 6[1111]=6[1111] \text {, } \\
& {[11] *[11]=\underline{[11]}+6[111]+6[1111](130)} \\
& {[11] *[2]=\{11,2\}_{2}+\{11,2\}_{3}+\{11,2\}_{4},} \\
& \{11,2\}_{2}=[11] \circ[2]=[2], \\
& \{11,2\}_{3}=\rho_{1}[11] \circ \rho_{1}[2]-\rho_{1}\left(\{11,2\}_{2}\right)=3[111] \circ[21]-[21]=2[21], \\
& \{11,2\}_{4}=\rho_{2}[11] \circ \rho_{2}[2]-\rho_{2}\left(\{11,2\}_{2}\right)-\rho_{1}\left(\{11,2\}_{3}\right)=6[1111] \circ[211]-[211]-2 \cdot 2[211]=[211], \\
& {[11] *[2]=\underline{[2]}+2[21]+[211]} \\
& {[2] *[2]=\{2,2\}_{2}+\{2,2\}_{3}+\{2,2\}_{4},} \\
& \{2,2\}_{2}=[2] \circ[2]=[11], \\
& \{2,2\}_{3}=\rho_{1}[2] \circ \rho_{1}[2]-\rho_{1}\left(\{2,2\}_{2}\right)=[21] \circ[21]-3[111]=3[3], \\
& \{2,2\}_{4}=\rho_{2}[2] \circ \rho_{2}[2]-\rho_{2}\left(\{2,2\}_{2}\right)-\rho_{1}\left(\{2,2\}_{3}\right)=[211] \circ[211]-6[1111]-3[31]=2[22], \\
& {[2] *[2]=\underline{[11]}+3[3]+2[22]}
\end{aligned}
$$

\subsection{Level $(1,4)$}

$$
\begin{array}{r}
{[1] *[1111]=\{1,1111\}_{4}+\{1,1111\}_{5},} \\
\{1,1111\}_{4}=\rho_{3}[1] \circ[1111]=4[1111] \circ[1111]=4[1111], \\
\{1,1111\}_{5}=\rho_{4}[1] \circ \rho_{1}[1111]-\rho_{1}\left(\{1,1111\}_{4}\right)=5[1111] \circ 5[1111]-5 \cdot 4[11111]=5[1111], \\
{[1] *[1111]=4[1111]+5[11111]}
\end{array}
$$




$$
\begin{gathered}
{[1] *[211]=\{1,211\}_{4}+\{1,211\}_{5},} \\
\{1,211\}_{4}=\rho_{3}[1] \circ[211]=4[1111] \circ[211]=4[211], \\
\{1,211\}_{5}=\rho_{4}[1] \circ \rho_{1}[211]-\rho_{1}\left(\{1,211\}_{4}\right)=5[1111] \circ 3[2111]-3 \cdot 4[2111]=3[2111], \\
{[1] *[211]=4[211]+3[2111]} \\
{[1] *[22]=\{1,22\}_{4}+\{1,22\}_{5},} \\
\{1,22\}_{4}=\rho_{3}[1] \circ[22]=4[1111] \circ[22]=4[22], \\
{[1,22\}_{5}=\rho_{4}[1] \circ \rho_{1}[22]-\rho_{1}\left(\{1,2\}_{4}\right)=5[1111] \circ[221]-4[221]=[221],} \\
[1] *[22]=4[22]+[221]] \\
{[1] *[31]=\{1,31\}_{4}+\{1,31\}_{5},} \\
\{1,31\}_{4}=\rho_{3}[1] \circ[31]=4[1111] \circ[31]=4[31], \\
{[1,\}_{5}=\rho_{4}[1] \circ \rho_{1}[31]-\rho_{1}\left(\{1,31\}_{4}\right)=5[1111] \circ 2[311]-2 \cdot 4[311]=2[311],} \\
{[1] *[31]=4[31]+2[311]} \\
\{1,4\}_{5}=\rho_{4}[1] \circ \rho_{1}[4]-\rho_{1}\left(\{1,4\}_{4}\right)=5[1111] \circ[41]-4[41]=[41], \\
{[1] *[4]=4[4]+[41]} \\
\end{gathered}
$$

\subsection{Level $(2,3)$}

$$
\begin{aligned}
& {[11] *[111]=\{11,111\}_{3}+\{11,111\}_{4}+\{11,111\}_{5},} \\
& \{11,111\}_{3}=\rho_{1}[11] \circ[111]=3[111], \\
& \{11,111\}_{4}=\rho_{2}[11] \circ \rho_{1}[111]-\rho_{1}\left(\{11,111\}_{3}\right)=6[1111] \circ 4[1111]-4 \cdot 3[1111]=12[1111], \\
& \{11,111\}_{5}=\rho_{3}[11] \circ \rho_{2}[111]-\rho_{2}\left(\{11,111\}_{3}\right)-\rho_{1}\left(\{11,111\}_{4}\right)= \\
& =10[11111] \circ 10[11111]-10 \cdot 3[11111]-5 \cdot 12[11111]=10[11111] \text {, } \\
& {[11] *[111]=3[111]+12[1111]+10[11111]} \\
& {[11] *[21]=\{11,21\}_{3}+\{11,21\}_{4}+\{11,21\}_{5},} \\
& \{11,21\}_{3}=\rho_{1}[11] \circ[21]=3[21], \\
& \{11,21\}_{4}=\rho_{2}[11] \circ \rho_{1}[21]-\rho_{1}\left(\{11,21\}_{3}\right)=6[1111] \circ 2[211]-2 \cdot 3[211]=6[211], \\
& \{11,21\}_{5}=\rho_{3}[11] \circ \rho_{2}[21]-\rho_{2}\left(\{11,21\}_{3}\right)-\rho_{1}\left(\{11,21\}_{4}\right)= \\
& =10[11111] \circ 3[2111]-3 \cdot 3[2111]-3 \cdot 6[2111]=3[2111], \\
& {[11] *[21]=3[21]+6[211]+3[2111]} \\
& {[11] *[3]=\{11,3\}_{3}+\{11,3\}_{4}+\{11,3\}_{5},} \\
& \{11,3\}_{3}=\rho_{1}[11] \circ[3]=3[3] \text {, } \\
& \{11,3\}_{4}=\rho_{2}[11] \circ \rho_{1}[3]-\rho_{1}\left(\{11,3\}_{3}\right)=6[1111] \circ[31]-3[31]=3[31], \\
& \{11,3\}_{5}=\rho_{3}[11] \circ \rho_{2}[3]-\rho_{2}\left(\{11,3\}_{3}\right)-\rho_{1}\left(\{11,3\}_{4}\right)= \\
& =10[11111] \circ[311]-3[311]-2 \cdot 3[311]=[311], \\
& {[11] *[3]=3[3]+3[31]+[311]}
\end{aligned}
$$




$$
\begin{gathered}
{[2] *[111]=\{2,111\}_{3}+\{2,111\}_{4}+\{2,111\}_{5},} \\
\{2,111\}_{3}=\rho_{1}[2] \circ[111]=[21], \\
\{2,111\}_{4}=\rho_{2}[2] \circ \rho_{1}[111]-\rho_{1}\left(\{2,111\}_{3}\right)=[211] \circ 4[1111]-2[211]=2[211], \\
\{2,111\}_{5}=\rho_{3}[2] \circ \rho_{2}[111]-\rho_{2}\left(\{2,111\}_{3}\right)-\rho_{1}\left(\{2,111\}_{4}\right)= \\
=[2111] \circ 10[11111]-3[2111]-3 \cdot 2[2111]=[2111], \\
{[2] *[111]=[21]+2[211]+[2111]} \\
\{2,21\}_{4}=\rho_{2}[2] \circ \rho_{1}[21]-\rho_{1}\left(\{2,21\}_{3}\right)=[211] \circ 2[211]-4 \cdot 3[1111]-3[31]=4[22]+3[31], \\
\{2,21\}_{5}=\rho_{3}[2] \circ \rho_{2}[21]-\rho_{2}\left(\{2,21\}_{3}\right)-\rho_{1}\left(\{2,21\}_{4}\right)= \\
=[2111] \circ 3[2111]-10 \cdot 3[11111]-3[311]-4[221]-2 \cdot 3[311]=2[221], \\
{[2] *[21]=3[111]+3[3]+4[22]+3[31]+2[221]} \\
\{2,21\}_{3}=\rho_{1}[2] \circ[21]=[21] \circ[21]=3[111]+3[3], \\
\{2] *[3]=\{2,3\}_{3}+\{2,3\}_{4}+\{2,3\}_{5}, \\
\{2,3\}_{3}=\rho_{1}[2] \circ[3]=[21] \circ[3]=2[21], \\
\{2,3\}_{4}=\rho_{2}[2] \circ \rho_{1}[3]-\rho_{1}\left(\{2,3\}_{3}\right)=[211] \circ[31]-2 \cdot 2[211]=4[4], \\
\{2,3\}_{5}=\rho_{3}[2] \circ \rho_{2}[3]-\rho_{2}\left(\{2,3\}_{3}\right)-\rho_{1}\left(\{2,3\}_{4}\right)= \\
=[2111] \circ[311]-3 \cdot 2[2111]-4[41]=[32], \\
{[2] *[3]=2[21]+4[4]+[32]}
\end{gathered}
$$

\begin{tabular}{|c|c|}
\hline$[1] *[5]$ & $=5[5]+[51]$ \\
\hline$[1] *[41]$ & $=5[41]+2[411]$ \\
\hline$[1] *[32]$ & $=5[32]+[321]$ \\
\hline$[1] *[311]$ & $=5[311]+3[3111]$ \\
\hline$[1] *[221]$ & $=5[221]+2[2211]$ \\
\hline$[1] *[2111]$ & $=5[2111]+4[21111]$ \\
\hline [1] * [11111] & $=5[11111]+6[111111]$ \\
\hline
\end{tabular}

Above tables reproduce the ones from s.2.4.4 of [26]. What follows are some new pieces of the ${ }^{*}$-multiplication tables.

\subsection{Level $(1,5)$}

7.15 Level $(3,3)$

$$
\begin{gathered}
{[111] *[111]=\underline{[111]}+12[1111]+30[11111]+20[111111]} \\
{[111] *[21]=\underline{[21]}+6[211]+9[2111]+4[21111]}
\end{gathered}
$$


$[21] *[21]=3[111]+3[3]+12[1111]+8[22]+9[31]+10[221]+6[311]+4[2211]$

$$
[21] *[3]=\underline{2[21]}+4[211]+8[4]+3[32]+4[41]+[321]
$$

$$
[3] *[3]=\{3,3\}_{3}+\{3,3\}_{4}+\{3,3\}_{5}+\{3,3\}_{6},
$$

$$
\{3,3\}_{3}=[3] \circ[3]=2[111]+[3],
$$

$\{3,3\}_{4}=\rho_{1}[3] \circ \rho_{1}[3]-\rho_{1}\left(\{3,3\}_{3}\right)=[31] \circ[31]-4 \cdot 2[1111]-[31]=8[22]+3[31]$,

$\{3,3\}_{5}=\rho_{2}[3] \circ \rho_{2}[3]-\rho_{2}\left(\{3,3\}_{3}\right)-\rho_{1}\left(\{3,3\}_{4}\right)=$ $=[311] \circ[311]-10 \cdot 2[11111]-[311]-8[221]-2 \cdot 3[311]=5[5]$, $\{3,3\}_{6}=\rho_{3}[3] \circ \rho_{3}[3]-\rho_{3}\left(\{3,3\}_{3}-\rho_{2}\left(\{3,3\}_{4}\right)-\rho_{1}\left(\{3,3\}_{5}\right)=\right.$ $=[3111] \circ[3111]-20 \cdot 2\left[1^{6}\right]-[3111]-8[2211]-3 \cdot 3[3111]-5[51]=2[33]$,

$$
[3] *[3]=2[111]+[3]+8[22]+3[31]+5[5]+2[33]
$$

\subsection{Selected products}

$$
\begin{array}{r}
{[3] *[33]=\{3,33\}_{6}+\{3,33\}_{7}+\{3,33\}_{8}+\{3,33\}_{9},} \\
\{3,33\}_{6}=\rho_{3}[3] \circ[33]=[3111] \circ[33]=2[3111]+2[33]+12[6], \\
\{3,33\}_{7}=\rho_{4}[3] \circ \rho_{1}[33]-\rho_{1}\left(\{3,33\}_{6}\right)= \\
=\left[31^{4}\right] \circ[331]-8\left[31^{4}\right]-2[331]-12[61]=8[43]+6[61], \\
\{3,33\}_{8}=\rho_{5}[3] \circ \rho_{2}[33]-\rho_{2}\left(\{3,33\}_{6}\right)-\rho_{1}\left(\{3,33\}_{7}\right)=5[53], \\
\{3,33\}_{9}=\rho_{6}[3] \circ \rho_{3}[33]-\rho_{3}\left(\{3,33\}_{6}\right)-\rho_{2}\left(\{3,33\}_{7}\right)-\rho_{1}\left(\{3,33\}_{8}\right)=3[333], \\
{[3] *[33]=2[33]+2[3111]+12[6]+8[43]+6[61]+5[53]+3[333]}
\end{array}
$$


Appendix III. The table of symmetric characters $\varphi_{R}(\Delta)$ at level 6

\begin{tabular}{|c|c||c|c|c|c|c|c|c|c|c|c|c|}
\hline$R \Delta$ & $d_{R}$ & {$[6]$} & {$[51]$} & {$[42]$} & {$[411]$} & {$[33]$} & {$[321]$} & {$[3111]$} & {$[222]$} & {$[2211]$} & {$[21111]$} & {$[11111]$} \\
\hline \hline$[6]$ & $\frac{1}{720}$ & 120 & 144 & 90 & 90 & 40 & 120 & 40 & $\mathbf{1 5}$ & 45 & 15 & 1 \\
\hline$[51]$ & $\frac{1}{144}$ & $-\mathbf{2 4}$ & 0 & -18 & 18 & -8 & 0 & 16 & $-\mathbf{3}$ & 9 & 9 & 1 \\
\hline$[42]$ & $\frac{1}{80}$ & 0 & -16 & 10 & -10 & 0 & 0 & 0 & $\mathbf{5}$ & 5 & 5 & 1 \\
\hline$[411]$ & $\frac{1}{72}$ & $\mathbf{1 2}$ & 0 & 0 & 0 & 4 & -12 & 4 & $-\mathbf{3}$ & -9 & 3 & 1 \\
\hline$[33]$ & $\frac{1}{144}$ & 0 & 0 & -18 & -18 & 16 & 24 & -8 & $-\mathbf{9}$ & 9 & 3 & 1 \\
\hline$[321]$ & $\frac{1}{45}$ & 0 & 9 & 0 & 0 & -5 & 0 & -5 & $\mathbf{0}$ & 0 & 0 & 1 \\
\hline$[3111]$ & $\frac{1}{72}$ & -12 & 0 & 0 & 0 & 4 & 12 & 4 & 3 & -9 & -3 & 1 \\
\hline$[222]$ & $\frac{1}{144}$ & 0 & 0 & -18 & 18 & 16 & -24 & -8 & 9 & 9 & -3 & 1 \\
\hline$[2211]$ & $\frac{1}{80}$ & 0 & -16 & 10 & 10 & 0 & 0 & 0 & -5 & 5 & -5 & 1 \\
\hline & $\frac{1}{144}$ & 24 & 0 & -18 & -18 & -8 & 0 & 16 & 3 & 9 & -9 & 1 \\
\hline
\end{tabular}

